\title{
A Critical Interaction between NR2B and MAGUK in L-DOPA Induced Dyskinesia
}

\author{
Fabrizio Gardoni, ${ }^{1 \star}$ Barbara Picconi, ${ }^{2 \star}$ Veronica Ghiglieri, ${ }^{2}$ Federica Pollii, ${ }^{1}$ Vincenza Bagetta, ${ }^{2}$ Giorgio Bernardi, ${ }^{2,3}$ \\ Flaminio Cattabeni, ${ }^{1}$ Monica Di Luca, ${ }^{1}$ and Paolo Calabresi ${ }^{2,4}$ \\ ${ }^{1}$ Center of Excellence on Neurodegenerative Diseases and Department of Pharmacological Sciences, University of Milan, 20133 Milan, Italy, ${ }^{2}$ Laboratorio di \\ Neurofisiologia, Fondazione Santa Lucia, Instituto di Ricovero e Cura a Carattere Scientifico, Centro Europeo di Ricerca sul Cervello, 00143 Rome, Italy, \\ ${ }^{3}$ Clinica Neurologica, Department of Neuroscience, University of Rome Tor Vergata, 00133 Rome, Italy, and ${ }^{4}$ Clinica Neurologica, Università di Perugia, \\ Ospedale Silvestrini, 06156 Perugia, Italy
}

\begin{abstract}
Abnormal function of NMDA receptor has been suggested to be correlated with the pathogenesis of Parkinson's disease (PD) as well as with the development of L-3,4-dihydroxyphenylalanine (L-DOPA)-induced dyskinesia. Here we show that NMDA receptor NR2 subunits display specific alterations of their subcellular distribution in striata from unilateral 6-hydroxydopamine-lesioned, L-DOPA-treated dyskinetic, and L-DOPA-treated nondyskinetic rats. Dyskinetic animals have significantly higher levels of NR2A subunit in the postsynaptic compartment than all other experimental groups, whereas NR2B subunit shows a significant reduction in both dopaminedenervated and dyskinetic rats. These events are paralleled by profound modifications of NMDA receptor NR2B subunit association with interacting elements, i.e., members of the membrane-associated guanylate kinase (MAGUK) protein family postsynaptic density-95, synapse-associated protein-97 and synapse-associated protein-102. Treatment of nondyskinetic animals with a synthetic peptide (TAT2B) able to affect NR2B binding to MAGUK proteins as well as synaptic localization of this subunit in nondyskinetic rats was sufficient to induce a shift of treated rats toward a dyskinetic motor behavior. These data indicate abnormal NR2B redistribution between synaptic and extrasynaptic membranes as an important molecular disturbance of the glutamatergic synapse involved in L-DOPA-induced dyskinesia.
\end{abstract}

Key words: Parkinson's disease; dyskinesia; 6-OHDA; NMDA receptor; MAGUK; rat

\section{Introduction}

Parkinson's disease (PD) is a progressive neurological disorder characterized by the degeneration of dopaminergic neurons projecting to the striatum. Accordingly, pharmacological dopamine (DA) replacement with chronic L-3,4-dihydroxyphenylalanine (L-DOPA) treatment represents the most effective therapeutic option (Lang and Lozano, 1998). Unfortunately, the development of disabling complications, including motor fluctuations and dyskinesia, represent a dramatic consequences of long-term L-DOPA treatment in PD patients (Obeso et al., 2000).

The subcellular organization as well as the functional interactions of glutamate receptors (GluRs) within the striatum appears to be crucial in the pathogenesis of PD as well as in the development of L-DOPA-induced dyskinesia (Hallett et al., 2005). NMDA receptor antagonists exert a beneficial effect in experimental models of PD (Nash et al., 2000; Loschmann et al., 2004),

\footnotetext{
Received July 14, 2005; revised Jan. 20, 2006; accepted Jan. 23, 2006.

This work was supported by the European Community (LSHM-CT-2004-511995, Synaptic Scaffolding Proteins Orchestrating Cortical Synapse Organisation during Development), by Fondo Integrativo Speciale per la Ricerca (P.C., M.D.L.), by Programmi di Ricerca Scientifica di Rilevante Interesse Nazionale 2005 (P.C.), by Fondo per gli Investimenti della Ricerca di Base 2001 (G.B., M.D.L.), and by Instituto di Ricovero e Cura a Carattere Scientifico (P.C., M.D.L.).

${ }^{*}$ F.G. and B.P. contributed equally to this work.

Correspondence should be addressed to Paolo Calabresi, Clinica Neurologica, Dipartimento di Specialità Medico Chirurgiche e Sanità Pubblica, Università di Perugia, 06156 Perugia, Italy. E-mail: calabre@unipg.it. DOI:10.1523/JNEUROSCI.5326-05.2006

Copyright $\odot 2006$ Society for Neuroscience $\quad 0270-6474 / 06 / 262914-09 \$ 15.00 / 0$
}

and they are also effective in blocking the development of L-DOPA-induced dyskinesias (Hadj Tahar et al., 2004; Wessell et al., 2004). At the molecular level, alterations of NMDA receptor subunit synaptic localization in striatum have been described in DA-denervated rats (Picconi et al., 2004) as well as in L-DOPAtreated dyskinetic monkeys (Hallett et al., 2005), even if the precise mechanisms regulating NMDA receptor subcellular trafficking and function in experimental parkinsonism are far from being elucidated.

NMDA receptors are enriched in a highly organized subcellular fraction, the postsynaptic density (PSD), in which binding of NMDA receptor subunits to other proteins is dynamically regulated (Gardoni et al., 2001; Sheng, 2001). Several molecular partners of NMDA receptors have been described, including scaffolding proteins and signaling elements (Kim and Sheng, 2004). In particular, members of the membrane-associated guanylate kinase (MAGUK) protein family interact through the PSD-95/ Discs large/zona occludens-1 (PDZ) domain with the C-terminal binding motifs present in NMDA receptor NR2 subunits. This interaction is instrumental for the early trafficking of NMDA receptor from the reticulum as well as for receptor stabilization at the synapse (Prybylowski and Wenthold, 2004). Although MAGUK members show similar specificities of protein interaction in vitro, they bind different sets of proteins in vivo. For instance, PSD-95 and synapse-associated protein-97 (SAP97) are 
preferentially associated with NR2A in vivo, whereas SAP102 is more associated with NR2B (Kim and Sheng, 2004).

A conclusive concept on possible perturbation of the major factors guiding NMDA receptors trafficking to, and functional organization in, the postsynaptic membranes in experimental parkinsonism and dyskinesia is still lacking. In this study, we evaluated the molecular composition of NMDA receptor complex at synapses in striata from sham, DA-denervated, and L-DOPA-treated dyskinetic and nondyskinetic animals. We investigated the abundance of NMDA receptor subunits in the Triton X-100-insoluble postsynaptic fraction (TIF), the interactions of NR2 subunits with MAGUK elements, as well as their calcium/ calmodulin-dependent kinase II (CaMKII)- and Tyr-dependent phosphorylation. Our data indicate NR2B subcellular redistribution between synaptic and extrasynaptic sites as a major element in the complex modification of glutamatergic synapse in L-DOPA-induced dyskinesia.

\section{Materials and Methods}

Animals. Adult male Wistar rats $(150-250 \mathrm{~g})$ were used for all of the experiments. Experiments were conducted in compliance with the European Council directive (86/609/EEC) for the use and care of laboratory animals.

6-Hydroxydopamine lesions. Deeply anesthetized rats were injected with 6-hydroxydopamine (6-OHDA) ( $8 \mu \mathrm{g} / 4 \mu \mathrm{l}$ saline containing $0.1 \%$ ascorbic acid) into the substantia nigra, at a rate of $0.38 \mu \mathrm{l} / \mathrm{min}$ (according to the atlas of Paxinos and Watson, 1986). Sham-operated rats were injected with vehicle at the same coordinates. Fifteen days later, the rats were tested with $0.05 \mathrm{mg} / \mathrm{kg}$ subcutaneous apomorphine, and contralateral turns to the lesion were counted for $40 \mathrm{~min}$. Only those rats that made at least 200 contralateral turns were used for biochemical and behavioral experiments. It has been demonstrated previously that rats meeting this screening criterion have $>95 \%$ depletion of striatal dopamine (Schwarting and Huston, 1996).

Chronic L-DOPA treatment and behavioral testing. At 2 months after the 6-OHDA lesion, rats started to receive twice daily intraperitoneal injections of $10 \mathrm{mg} / \mathrm{kg}$ L-DOPA plus $7.5 \mathrm{mg} / \mathrm{kg}$ benserazide, or physiological saline, for 12-16 d. L-DOPA-induced abnormal involuntary movements (AIMs) were recorded on alternate days three times per week using a validated rat AIM scale, as described previously (Cenci et al., 1998; Lundblad et al., 2002; Picconi et al., 2003). Briefly, rat AIMs were classified into three subtypes: axial AIMs (i.e., dystonic posturing of the upper part of the body toward the side contralateral to the lesion), limb AIMs (i.e., abnormal movements of the forelimb contralateral to the lesion), and orolingual AIMs (i.e., empty jaw movements and tongue protrusion). Each of these subtypes was scored on a severity scale from 0 to 4: 1, present during less than half of the observation time ( $1 \mathrm{~min}$ ); 2 , present during more than half of the time; 3 , present all the time but was arrested by extern stimuli; 4 , present all the time without possibility to arrest it. Ratings were performed at 20-180 min after L-DOPA injection.

Each rat could reach a theoretical maximum AIM score of 80 in one session; each session consisted of five monitoring periods, with a maximal score in each period of 16 , and the total AIM score for each session was obtained by summing the monitoring period scores. The rats that reach more than AIM score of 25 per session were included in the dyskinetic group.

The effects of cell-permeable TAT peptide fused to the last C-terminal nine amino acids of NR2B (TAT2B) on the AIM response to chronic L-DOPA treatment was assessed by intrastriatal injection of the peptide in 6-OHDA-lesioned rats, sham-operated animals, and L-DOPA-treated rats that did or did not display dyskinesias.

Each experimental group (sham, 6-OHDA, dyskinetic, and nondyskinetic rats), after 2 weeks of L-DOPA-treatment, received three consecutive injections (one per day) with TAT2B (500 $\mu \mathrm{M})$ (Aarts et al., 2002) into the striatum ipsilateral to the lesion at a rate of $0.5 \mu \mathrm{l} / \mathrm{min}$ (total volume of $1 \mu \mathrm{l}$ ) using the following stereotaxic coordinates: anteroposterior, +0.7 ; lateral, +4 ; ventrodorsal, -4.6 . TAT2B $(\Delta \mathrm{SDV})(500 \mu \mathrm{M})$ peptide lacking the last three amino acids of the NR2B subunit was used as control peptide. L-DOPA treatments were administered $1 \mathrm{~h}$ after TAT2B or TAT2B $(\Delta S D V)$ intrastriatal injection. To analyze the effect of TAT2B and TAT2B $(\Delta \mathrm{SDV})$ on the L-DOPA-induced dyskinesias, rating of AIMs were performed each day of peptide injection $20 \mathrm{~min}$ after L-DOPA administration. One hour after intrastriatal administration of TAT2B, the same time course adopted for the behavioral scoring of the other experimental groups was used. Three consecutive AIM tests before the injection of these specific peptides were compared with the last three AIM ratings performed after each injection.

Western blot analysis. Western blot analysis was performed in striatal tissue from the whole corpus striatum. Subcellular fractionation was performed as reported previously with minor modifications (see Fig. 1a) (Gardoni et al., 2001). Striata were homogenized in $0.32 \mathrm{M}$ ice-cold sucrose containing the following (in mM): 1 HEPES, $1 \mathrm{MgCl}_{2}, 1$ EDTA, 1 $\mathrm{NaHCO}_{3}$, and 0.1 PMSF, at pH 7.4, in the presence of a complete set of proteases inhibitors (Complete; Roche Diagnostics, Basel, Switzerland) and phosphatases inhibitors (Sigma, St. Louis, MO). The homogenized tissue was centrifuged at $1000 \times g$ for $10 \mathrm{~min}$. The resulting supernatant (S1) was centrifuged at $3000 \times g$ for 15 min to obtain a crude membrane fraction (P2 fraction). The pellet was resuspended in 1 mM HEPES plus CompleteTM in a glass-glass potter and centrifuged at $100,000 \times g$ for $1 \mathrm{~h}$. The pellet (P3) was resuspended in buffer containing $75 \mathrm{~mm} \mathrm{KCl}$ and $1 \%$ Triton X-100 and centrifuged at $100,000 \times g$ for $1 \mathrm{~h}$. The supernatant was stored and referred as Triton X-100-soluble fraction (TSF) (S4). The final pellet (P4) was homogenized in a glass-glass potter in $20 \mathrm{~mm}$ HEPES. Then, an equal volume of glycerol was added, and this fraction, referred as TIF, was stored at $-80^{\circ} \mathrm{C}$ until processing. TIF was used instead of the classical PSD because the amount of the starting material was very limited. The protein composition of this preparation was, however, carefully tested for the absence of presynaptic markers (i.e., synaptophysin) (Gardoni et al., 2001) (see Fig. 1b). Similar protein yield was obtained in TIF purified from striata of all experimental groups, and the same amount of TIF protein was applied to SDS-PAGE and electroblotted for all samples. Nitrocellulose papers were blocked with $10 \%$ albumin in Tris-buffered saline (TBS) and then incubated for $2 \mathrm{~h}$ at room temperature with the primary antibodies: NR1 (diluted 1:1000), NR2A (diluted 1:1000), NR2B (diluted 1: 1000), p1303ser-NR2B (diluted 1:750), p1472Tyr-NR2B (diluted 1:1000), GluR1 (diluted 1:1000), PSD-95 (diluted 1:2000), SAP97 (diluted 1:1000), SAP102 (diluted 1:1000), $\alpha$ CaMKII (diluted 1:3000), cAMP response element-binding protein (CREB) (diluted 1: 1000), and phosphorylated CREB (pCREB) (diluted $1: 750$ ) in $3 \%$ albumin in TBS. After extensive rinsing in TBS/0.1\% Tween 20, the nitrocellulose papers were then incubated with horseradish peroxidase-conjugated secondary antibodies [goat anti-rabbit, for polyclonal antibodies, diluted 1:10,000 (Pierce, Rockford, IL); goat antimouse, for monoclonal antibodies, diluted 1:20000 (Pierce)], and then the antigen-antibody complex was revealed by enhanced chemiluminescence (ECL; Amersham Biosciences, Little Chalfont, UK).

Immunoprecipitation experiments. Aliquots of striatal homogenates and TIF were incubated overnight at $4^{\circ} \mathrm{C}$ in buffer $\mathrm{A}$, containing the following: $200 \mathrm{~mm} \mathrm{NaCl}, 10 \mathrm{~mm}$ EDTA, $10 \mathrm{~mm} \mathrm{Na}_{2} \mathrm{HPO}_{4}, 0.5 \% \mathrm{NP}-40$, and $0.1 \%$ SDS with antibodies against PSD-95, NR2A, or NR2B subunits of NMDA receptor. Protein A-agarose beads (Santa Cruz Biotechnology, Santa Cruz, CA), washed in the same buffer, were added, and incubation continued for $2 \mathrm{~h}$. The beads were collected by centrifugation and washed five times with buffer A, sample buffer for SDS-PAGE was added, and the mixture was boiled for $5 \mathrm{~min}$. Beads were pelleted by centrifugation, and supernatants were applied to $6 \%$ SDS-PAGE.

Antibodies. The following antibodies were used: mouse $\alpha$ CaMKII antibody and polyclonal GluR1, NR2A, and NR2B antibodies were purchased from Chemicon (Temecula, CA). Polyclonal SAP97 and SAP102 antibodies and monoclonal PSD-95 antibody were purchased from Affinity BioReagents (Golden, CO). Monoclonal SAP97 antibody was purchased from StressGen (Victoria, British Columbia, Canada). Polyclonal p1472Tyr-NR2B, CREB, and p133-CREB antibodies were purchased from Calbiochem (Nottingham, UK). Polyclonal p1303Ser-NR2B antibody was purchased from Upstate (Waltham, MA). Monoclonal NR1 antibody was purchased from Zymed (San Francisco, CA). 
Data analysis and statistical evaluation. Quantitation of Western blot analysis was performed by means of computer-assisted imaging (Quantity-One System; Bio-Rad, Hercules, $\mathrm{CA})$, and statistical evaluations were performed according to one-way ANOVA, followed by Bonferroni's test for post hoc comparison.

\section{Results}

In the present study, we used therapeutic doses of L-DOPA (10 $\mathrm{mg} / \mathrm{kg}$ methyl L-DOPA combined with $7.5 \mathrm{mg} / \mathrm{kg}$ benserazide, twice daily), which induced a gradual development of AIMs (Picconi et al., 2003) in $~ 50 \%$ of the rats. Before the onset of L-DOPA treatment, these two groups of subjects exhibited the same degree of impairment in motor performances and spontaneous forelimb use (data not shown). After $12 \mathrm{~d}$ of L-DOPA treatment, the two subpopulations were distinguished in response to L-DOPA based on the degree of AIM development (see Materials and Methods), which precluded fine assessment of motor skills in the dyskinetic group (Picconi et al., 2003).

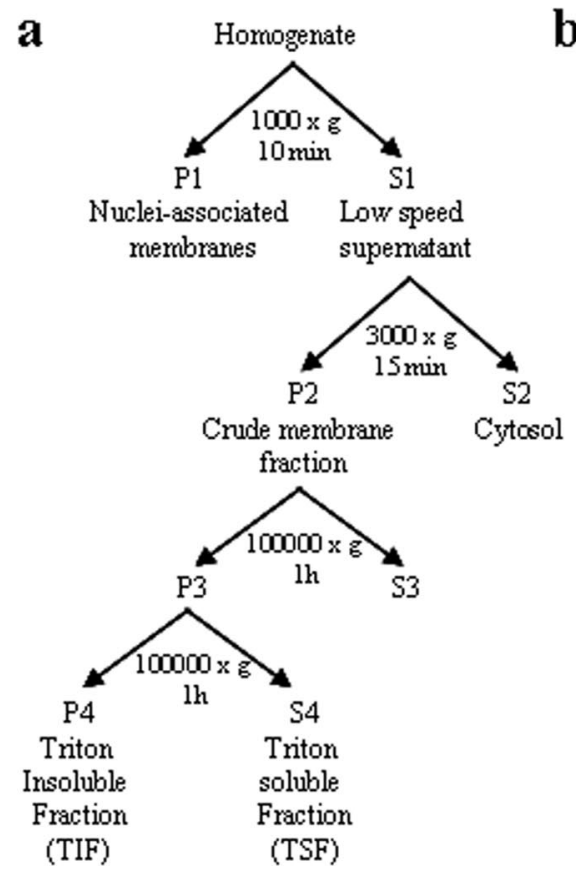

b

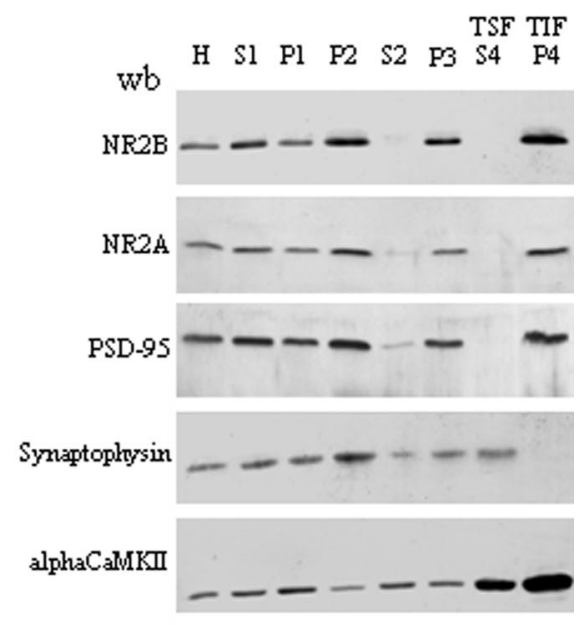

Figure 1. $a$, Characterization of biochemical fractionation method used in the present study. The procedure for the subcellular fractionation is described in Materials and Methods. $\boldsymbol{b}$, The isolated biochemical fractions from striatal tissues were separated by SDS-PAGE, and the blots were probed with antibodies against NR2B, NR2A, PSD-95, synaptophysin, and $\alpha$ CaMKII. wb, Western blot.

\section{Characterization of}

subcellular compartments

A biochemical fractionation method (Fig. 1a) was used to purify subcellular compartments from striatal tissues, and the effectiveness of the procedure was evaluated by Western blotting analysis for protein markers for subcellular compartments (Fig. 1b). PSD-95 and NMDA receptor subunits NR2A and NR2B were found enriched in crude membrane fraction (P2), in the high speed pellet (P3), and in TIF. These proteins were also found, even if with a lower abundance, in total homogenate $(\mathrm{H})$, in low-speed supernatant (S1), and in nuclei-associated membrane (P1). However, the NMDA subunits NR2A and NR2B as well as PSD-95 were not detectable in the cytosolic (S2) or TSF. The localization of NMDA receptor subunits NR2A and NR2B in striatal tissue was similar to what was reported previously in other brain regions (Gardoni et al., 2003b). As expected, synaptophysin, a synaptic vesicle membrane protein enriched in the presynaptic terminals, was found to be concentrated in the P2 crude membrane fraction and in the TSF but was not detected in the TIF. CaMKII was present in all fractions but enriched in TSF and TIF as reported previously (Gardoni et al., 2003a).

\section{Effect of 6-OHDA lesioning and subsequent chronic L-DOPA treatment on NMDA receptor \\ subunits in rat striatum}

NMDA receptor subunit protein levels were examined by means of Western blot analysis performed in homogenate and TIF (Picconi et al., 2004) prepared from striata of control, 6-OHDA, and L-DOPA-treated nondyskinetic and dyskinetic rats. Similar protein yield was obtained in TIF purified from all groups, and the same amount of TIF proteins was applied to SDS gel and electroblotted. No difference was found in the expression level of any tested NMDA subunit in the homogenate fraction (Fig. 2, left panels). As reported previously (Picconi et al., 2004), levels of NR2B were specifically reduced in TIF purified from 6-OHDA rats when compared with sham rats in the absence of parallel alterations of NR1 and NR2A in the same samples (Fig. 2, right panels).

More complex alterations of the NMDA receptor in the TIF were found as a direct consequence of chronic L-DOPA treatment. Profound differences in NMDA receptor composition in the TIF postsynaptic compartment were found between L-DOPA-treated dyskinetic and nondyskinetic rats. Dyskinetic animals were characterized by a significant increase in the NR2A immunostaining in the TIF that was accompanied by a concomitant decrease of NR2B protein level in the same subcellular compartment (Fig. 2, right panels). No alteration in NR1 immunostaining was present in either dyskinetic and nondyskinetic rats when compared with controls, suggesting a specific modification of NR2-type regulatory subunits induced by chronic L-DOPA treatment.

\section{Effect of 6-OHDA lesioning and subsequent chronic L-DOPA treatment on MAGUK-NMDA receptor subunit functional interaction}

It is known that the functional properties of NMDA receptors are strictly correlated to their subunit composition as well as by the composition of the complex formed with NMDA receptor interacting proteins, i.e., MAGUK family members. Recent data showed that interactions of NMDA receptor subunits NR2A/B with SAP102 (Sans et al., 2003) and SAP97 (Gardoni et al., 2003a) are involved in the NMDA receptor trafficking to synaptic sites in neurons. On this ground, we tested whether alterations in synaptic abundance of NR2A and NR2B found in experimental animals were attributable to abnormalities of their interaction with MAGUK members. Analysis of PSD-95, SAP97, and SAP102 in postsynaptic TIF revealed a significant reduction of the three proteins in 6-OHDA rats compared with sham-operated rats (Fig. 3, right panels). SAP97 and SAP102 protein levels were rescued to control values and beyond in chronically L-DOPAtreated dyskinetic and nondyskinetic animals, respectively (Fig. 


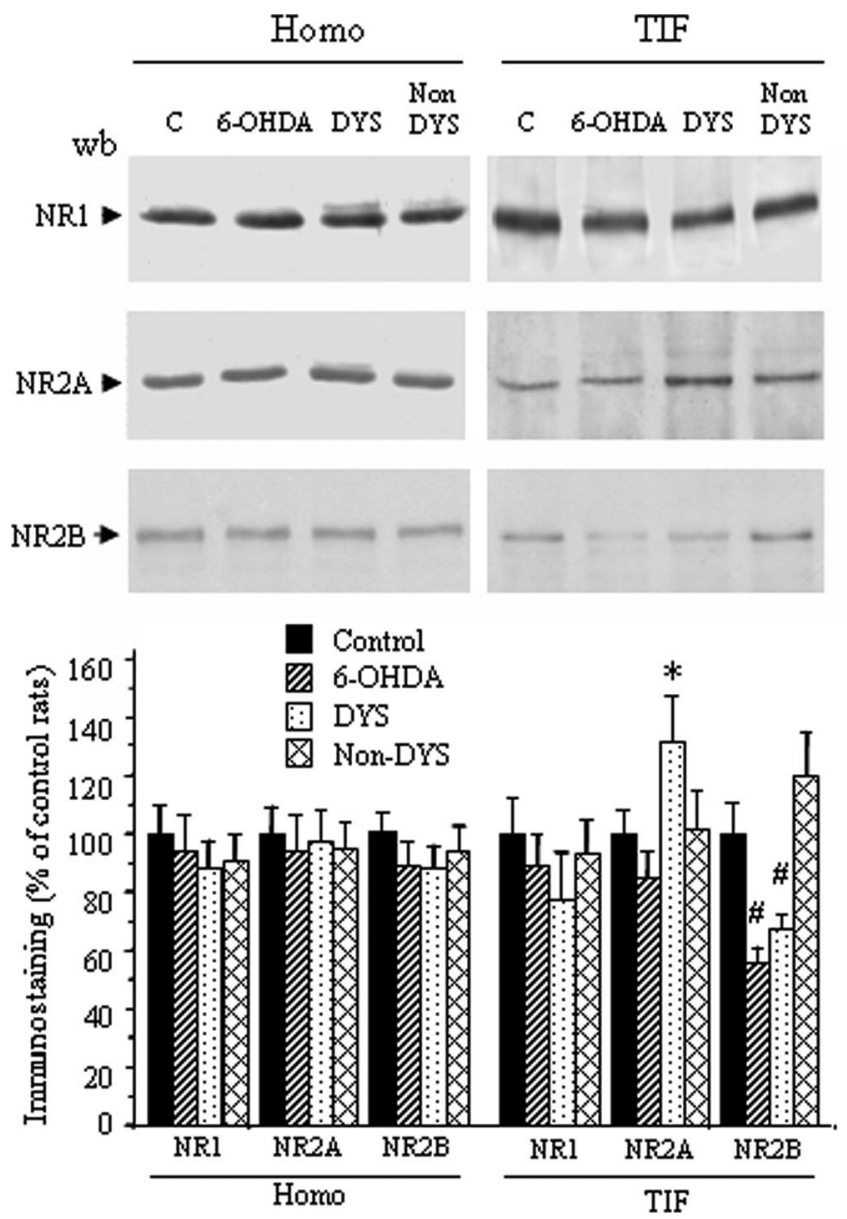

Figure 2. Effects of 6-OHDA lesioning and subsequent L-DOPA treatment on NMDA receptor subunit protein levels in rat striatum. 6-OHDA-lesioned animals were treated chronically with L-DOPA and divided into two groups based on the presence (DYS) or absence (Non DYS) of dyskinesia. Striatal homogenates (Homo; left) and TIF (right) from sham and 6-OHDA-lesioned dyskinetic and nondyskinetic animals were analyzed by Western blot (wb) analysis with NR1, NR2A, and NR2B antibodies. The same amount of protein was loaded per lane (NR2A,${ }^{*} p<$ 0.05 , dyskinesia vs control; $\mathrm{NR2B},{ }^{\#} p<0.01$, dyskinesia vs control and $6-0 \mathrm{HDA}$ vs control; $n=$ 8 for each group). C, Control.

3, right panels). Conversely, PSD-95 abundance was not completely restored by L-DOPA, and no significant difference was found between dyskinetic and nondyskinetic animals. No changes were observed in the whole homogenate samples, suggesting a specific redistribution to the postsynaptic compartment (Fig. 3, left panels). Because interactions with MAGUK family members are responsible for the correct trafficking/localization of NR2 subunits to the synapse (Prybylowski and Wenthold, 2004), we checked for NR2A and NR2B interaction with SAP97 and SAP102 by coimmunoprecipitation studies. Anti-NR2A and anti-NR2B immunoprecipitates were challenged in Western blotting with NR2A or NR2B, PSD-95, SAP97, and SAP102 antibodies (Fig. 4). No alteration of binding of any member of MAGUK family to NR2A was found in any experimental group (Fig. 4b). Conversely, Figure $4 a$ shows that the binding of NR2B to SAP102 was significantly reduced both in DA-denervated and L-DOPA-treated dyskinetic rats. Notably, this pattern perfectly superimposed the NR2B protein levels in TIF (Fig. 2), confirming the already suggested critical role of SAP102-NR2B complex formation for the correct delivery of NR2B to the synapse (Sans et al., 2003). In addition, an altered binding of both PSD-95 and SAP97 to NR2B was also observed (Fig. $4 a$ ), reduced in 6-OHDA

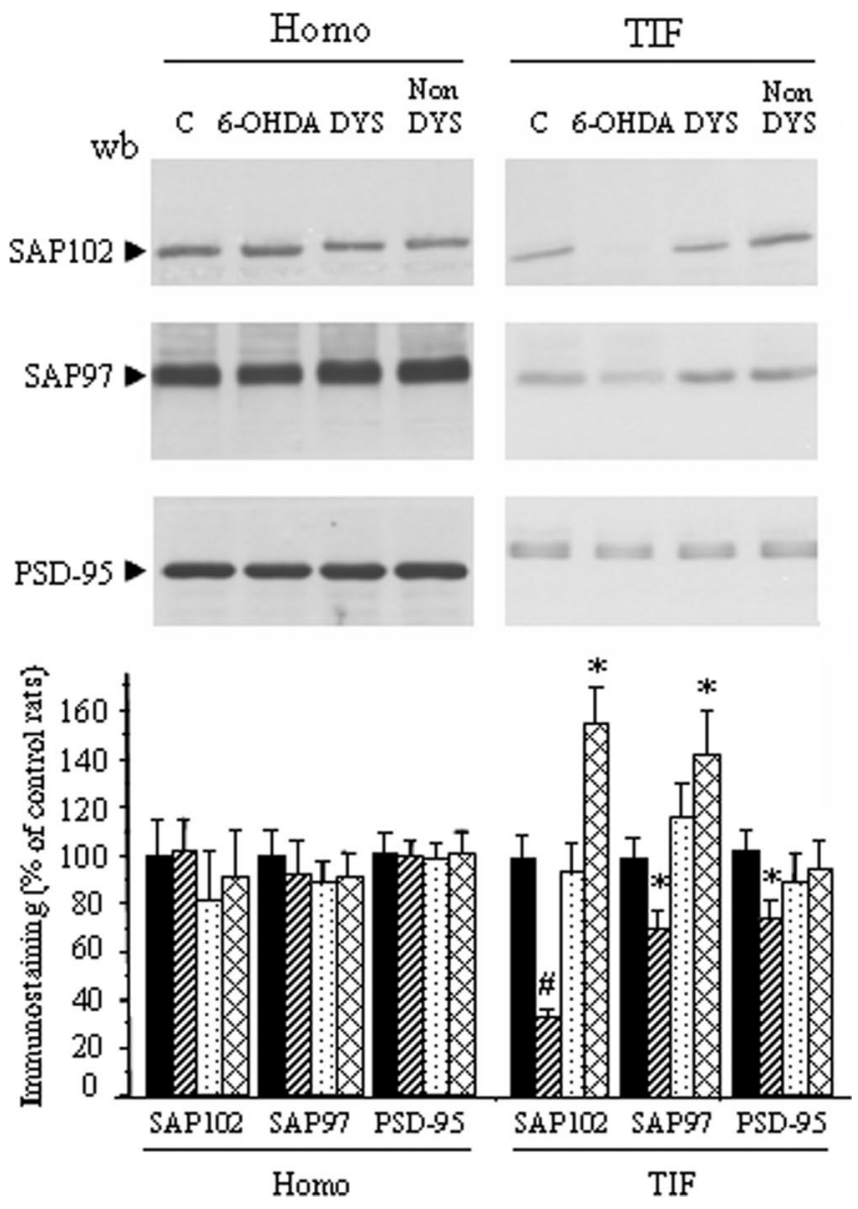

Figure 3. Striatal homogenates (Homo; left) and TIF (right) from sham and 6-OHDAlesioned animals in the presence (DYS) and absence (Non DYS) of dyskinesia were analyzed by Western blot (wb) analysis with PSD-95, SAP97, and SAP102 antibodies. The same amount of protein was loaded per lane. Histograms show the quantification of Western blotting for MAGUK proteins performed in homogenate and TIF fractions. Data are normalized as percentage of PSD-95, SAP97, and SAP102 immunostaining in control (C) rats ( ${ }^{*} p<0.05$, SAP97 and PSD-95 6-OHDA vs control, SAP97 and SAP102 nondyskinesia vs control; ${ }^{*} p<0.005$, SAP102 $6-\mathrm{OHDA}$ vs control; $n=8$ for each group).

rats and increased in nondyskinetic animals, suggesting an overall change of NMDA-NR2B subunit interaction with MAGUK proteins in experimental parkinsonism in rat. The binding of MAGUK proteins to NR2A/B subunits in the coimmunoprecipitation experiments was not attributable to an incomplete solubilization of striatal lysates, because another PSD protein, i.e., GluR1, was not detected in the precipitated material (Fig. 4a,b) (Gardoni et al., 1998).

\section{Ser and Tyr phosphorylation of NR2B after DA denervation} and chronic L-DOPA treatment

A direct phosphorylation of the NR2B subunit by both CaMKII (Omkumar et al., 1996; Gardoni et al., 1998; Strack and Colbran, 1998) and tyrosine kinases (Dunah and Standaert, 2001) in specific phospho-sites has been described to be mandatory for the dynamic regulation of NR2B trafficking/turnover. Of relevance, NR2B phosphorylation has been shown to be mainly restricted to a synaptic fraction and to play a role in the alterations of the NMDA receptor complex in DA-denervated striata (Menegoz et al., 1995; Oh et al., 1999; Dunah et al., 2000; Picconi et al., 2004). Thus, we monitored NR2B Ser and Tyr phosphorylation using 


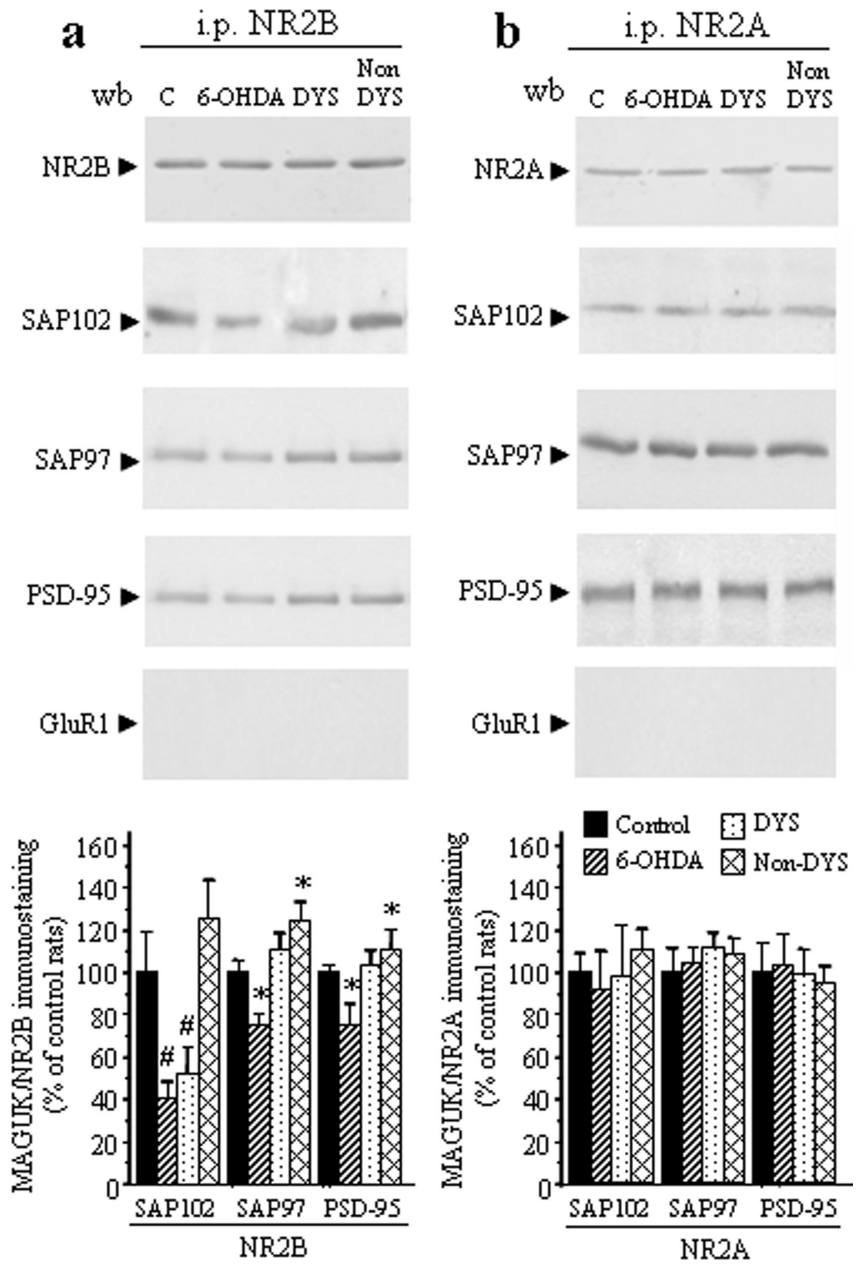

Figure 4. Striatal homogenates from sham, 6-OHDA, and L-DOPA-treated dyskinetic (DYS) and nondyskinetic (Non DYS) animals were immunoprecipitated with NR2B $(\boldsymbol{a})$ or NR2A $(\boldsymbol{b})$ polyclonal antibodies; Western blot analysis was performed in the immunoprecipitated material with NR2B, NR2A, SAP102, SAP97, PSD-95, and GluR1 antibodies. Histograms show the quantification of Western blotting for MAGUK proteins performed in the immunoprecipitated material. Data are normalized for total NR2A or NR2B staining in the immunocomplex $\left({ }^{\#} p<\right.$ 0.01, SAP102/NR2B, 6-OHDA vs control and dyskinesia vs control; ${ }^{*} p<0.05$, PSD-95/NR2B and $\mathrm{SAP97/NR2B}$, nondyskinesia vs control and 6-OHDA vs control; $n=6$ in each group). C, Control.

two phospho-specific antibodies raised against the CaMKIIdependent Ser1303 and the tyrosine kinase Tyr1472 phosphosites in the C-terminal domain of the NR2B subunit (Fig. $5 a, b$ ). Western blotting for total NR2B was always run in parallel samples. The relative amount of phosphorylation of the two sites was measured following two different approaches. First, the immunoreactivity of the bands corresponding to the phosphorylated proteins was quantified as percentage of immunoreactivity of the same bands in control rats (Fig. $5 c, d$ ), and second, they were adjusted for the abundance of the total NR2B subunit (Fig. 5e,f). No main modifications of both p1303Ser-NR2B and p1472TyrNR2B immunostaining was detected in the total striatal homogenates (Fig. $5 a$ ). Interestingly, the pattern of NR2B phosphospecific antibodies in TIF fractions was similar to the altered level of total NR2B, being significantly reduced in 6-OHDA and dyskinetic animals (Figs. 1, 5b-d) (Oh et al., 1999) when compared with control and nondyskinetic animals; thus, no significant difference was found in the ratio of phospho-NR2B/total NR2B in all experimental groups (Fig. 5e,f)
Subcellular redistribution of NR2B between synaptic and extrasynaptic sites in L-DOPA-treated nondyskinetic rats To study in more detail the subcellular distribution of NR2B in L-DOPA-treated animals, Western blotting analysis for this subunit was performed also in the $\mathrm{P} 2$ crude membrane fraction and in the TSF (see Materials and Methods). No alteration of NR2B protein levels was found in the $\mathrm{P} 2$ crude membrane fraction (Fig. $6 a$ ). Notably, analysis of NR2B levels in the TSF showed a high protein level in 6-OHDA and dyskinetic animals, whereas NR2B was barely detectable in the TSF from nondyskinetic rats and was not found in control animals (Figs. 1, 6a). These results suggest a redistribution of NR2B from insoluble to soluble membrane fractions in the denervated as well as in the dyskinetic animal, most likely from synaptic to extrasynaptic sites. To test this hypothesis, we performed Western blotting analysis for CREB and pCREB from striatal homogenates (Fig. 6b). In fact, it is known that synaptic NMDA receptors containing the NR2B subunits promote nuclear signaling to CREB, whereas extrasynaptic complexes antagonize nuclear signaling to CREB (Hardingham et al., 2002). Figure $6 b$ shows that levels of pCREB are dramatically reduced in both 6-OHDA and L-DOPA-treated dyskinetic animals compared with the other experimental groups, confirming the indication of an increased number of extrasynaptic NR2Bcontaining NMDA receptors in 6-OHDA and dyskinetic animals.

\section{Synaptic localization of NR2B subunit in chronic L-DOPA-} treated animals influences dyskinetic motor behavior The above described experiments suggest that NR2B levels at synaptic sites could represent a key element in the complex modification of the glutamatergic synapse in L-DOPA-induced dyskinesia. No alteration of NR2B localization was found in L-DOPAtreated nondyskinetic animals. It is well established that interactions of NR2B subunit C-terminal domain with the PDZ domains of MAGUK proteins play an important role in the regulation of NMDA receptor localization at synaptic sites (Sheng, 2001; Prybylowski and Wenthold, 2004). Based on these observations, we tested whether motor behavior in nondyskinetic rats was affected by acute dissociation of the MAGUK-NR2B subunit interaction through injection of a cell-permeable TAT peptide fused to the last C-terminal nine amino acids of NR2B (TAT2B) (Aarts et al., 2002; Lim et al., 2003). Treatments with $\mathrm{TAT} 2 \mathrm{~B}(\Delta \mathrm{SDV})$ peptide (lacking the last $\mathrm{C}$-terminal three amino acids of NR2B) were also performed as controls.

After 2 weeks of L-DOPA treatment, each experimental group (sham, 6-OHDA, dyskinetic, and nondyskinetic rats) received three consecutive injections with TAT2B $(500 \mu \mathrm{M})$ or $\mathrm{TAT} 2 \mathrm{~B}(\Delta \mathrm{SDV})(500 \mu \mathrm{M}), 1 \mathrm{~h}$ before L-DOPA administration, into the striatum ipsilateral to the lesion. First, we verified whether TAT2B could perturb NR2B-MAGUK protein complexes by coimmunoprecipitation experiments performed in homogenate of nondyskinetic striata in the presence or absence of TAT2B or TAT2B $(\Delta S D V)$ treatments. Treatment with TAT2B was able to reduce significantly NR2B but not NR2A coprecipitation with PSD-95 (Fig. $7 c)(-44.4 \pm 6.4 \%$; $p<0.01$, NR2B nondyskinetic plus TAT2B vs nondyskinetic), demonstrating that this approach is useful to mimic in nondyskinetic animals the low MAGUK-NR2B interaction found in dyskinetic rats. As expected, no effect on NR2B interaction with PSD-95 was induced by the TAT2B $(\Delta \mathrm{SDV})$ peptide (Fig. $7 c)[-3.9 \pm 8.1 \%$; $p>0.05$ NR2B nondyskinetic plus TAT2B $(\Delta \mathrm{SDV})$ vs nondyskinetic]. Similar effects on interaction with NR2B were obtained in coimmunoprecipitation experiments performed with SAP102 and SAP97 antibodies instead of PSD-95 antibody (data not 


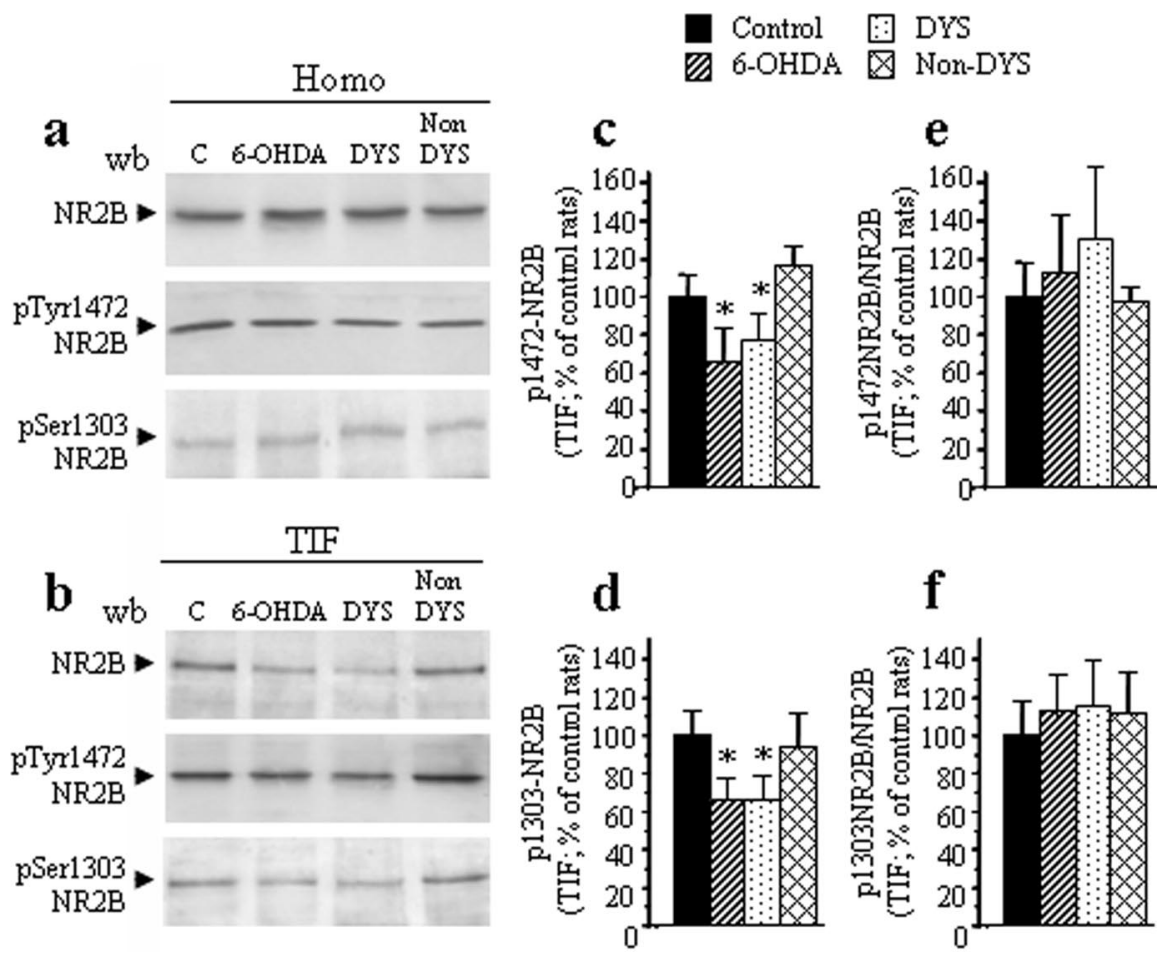

Figure 5. Striatal homogenates (Homo; $\boldsymbol{a}$ ) and TIF ( $\boldsymbol{b}$ ) from sham, 6-OHDA-lesioned, dyskinetic (DYS), and nondyskinetic (Non DYS) animals were analyzed by Western blot (wb) with NR2B, p1472Tyr-NR2B, and p1303Ser-NR2B antibodies. Histograms show the quantification of Western blotting in the TIF performed as pTyr1472-NR2B (c) and pSer1303-NR2B (d) immunostaining or normalized for total NR2B $(\boldsymbol{e}, \boldsymbol{f})$ immunoreactivity in the TIF $\left({ }^{*} p<0.05\right.$, p1472-NR2B and p1303-NR2B, 6-OHDA vs control and dyskinesia vs control; $n=6$ in each group). C, Control.

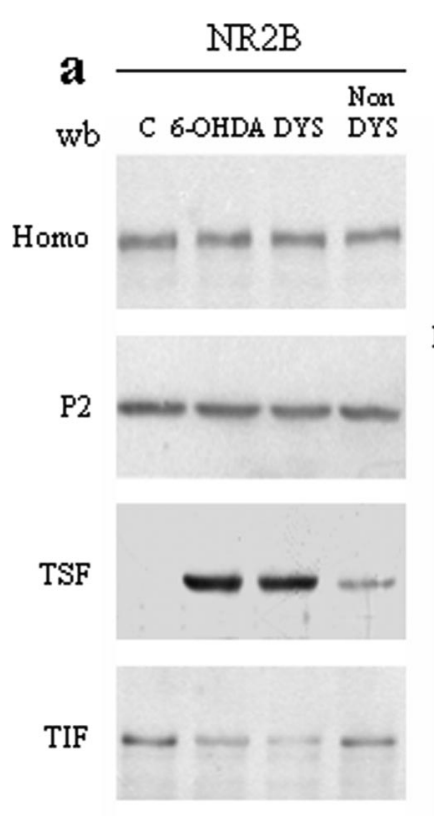
(Fig. 7d). In particular, nondyskinetic rats treated with uncoupling peptide showed TAT2B-induced dyskinetic behavior since the first day of treatment, reaching $\sim 50 \%$ of the intensity observed in untreated dyskinetic animals, as shown in Figure $7 d$ (nondyskinetic plus TAT2B AIM, $12.73 \pm 3.784$; dyskinetic AIMs, $26.70 \pm 1.787 ; p<0.05$, nondyskinetic vs nondyskinetic plus TAT2B and nondyskinetic plus TAT2B $(\Delta \mathrm{SDV})$ vs nondyskinetic plus TAT2B; $p<0.01$, dyskinetic vs nondyskinetic).

\section{Discussion}

In the present study, we performed a comprehensive analysis of NMDA receptor complex in striata from DA-denervated rats and subsequently chronically treated with L-DOPA. Hemiparkinsonian rats treated with L-DOPA were divided into two groups: one showing motor improvement without dyskinesia, and the other developing debilitating dyskinesia in response to the treatment (Picconi et al., 2003).

We show that NR2B subcellular redistribution from synaptic to extrasynaptic sites represents the key element in the complex modifications of the glutamatergic synapse in L-DOPA-induced dyskinesia. No alteration of NR2B synaptic localization was found in L-DOPA-treated nondyskinetic animals. In addition, dyskinetic animals have significantly higher levels of NR2A subunit in the postsynaptic compartment than in all other experimental groups.

These events are paralleled, and most probably triggered, by profound modifications of NMDA receptor NR2B subunit association with known interacting elements, i.e., members of the MAGUK protein family. On this view, we demonstrate that a treatment able to affect the normal synaptic localization of NR2B

Figure 6. a, Striatal homogenates (Homo), crude membrane fractions (P2), TSF, from control (C), 6-OHDA-lesioned, dyskinetic (DYS), and nondyskinetic (Non DYS) rats were analyzed by Western blot (wb) analysis with NR2B antibody. $\boldsymbol{b}$, Striatal homogenates (Homo) from sham, 6-OHDA-lesioned, dyskinetic (DYS), and nondyskinetic (Non DYS) animals were analyzed by Western blot analysis with CREB (top) and pCREB (bottom) antibodies. Histogram shows quantification of CREB phosphorylation in the striatal homogenate in the various experimental groups. Data are normalized for total CREB immunoreactivity in the respective samples ( $\#<0.01$, pCREB/CREB 6-OHDA vs control and dyskinesia vs control; $n=8$ in each group).

shown), as well as in experiments perand dyskinetic striata in the presence or shown).
Western blotting analysis was performed to test the consequences of TAT2B treatment on NR2B localization
in the TIF. Figure $7, a$ and $b$, shows that treatment with TAT2B was capable to reduce dramatically NR2B protein levels in
TIF in nondyskinetic animals $(p<0.01$, NR2B nondyskinetic plus TAT2B vs non-
dyskinetic). No effect of TAT2B was detected in Western blotting performed cating that the peptide did not affect NR2B expression (Fig. 7a, left). No effect on NR2B localization in the TIF was induced by treatment with $\mathrm{TAT} 2 \mathrm{~B}(\Delta \mathrm{SDV})$
control peptide (Fig. $7 a, b)$. In addition, no modification of both NR2A and PSD-95 staining in the TIF was induced
by TAT2B treatment (Fig. $7 a, b$, right). The injection of TAT2B did not produce 6-OHDA-lesioned rats and dyskinetic anmals (data not shown).

Interestingly, dissociation of the
subunit interaction through the injections of TAT2B coupled to L-DOPA administration showed the induction of AIMs in the nondyskinetic rats (1) 
subunit, by disrupting interaction of this subunit with MAGUK proteins, is sufficient to induce a dyskinetic motor behavior in L-DOPA-treated nondyskinetic animals.

In the last few years, many groups have reported alterations in both the composition and the phosphorylation state of NMDA receptors in experimental parkinsonism (Menegoz et al., 1995; Oh et al., 1999; Dunah et al., 2000; Picconi et al., 2004). Our results confirm and extend these data, demonstrating not only an altered compartmentalization of the NMDA receptor subunits in either 6-OHDA or dyskinetic rats but also a complex alteration of NMDA receptorassociated proteins, such as MAGUK members. These data suggest that chronic L-DOPA regulates NMDA subunit trafficking and binding to cargo proteins but does not alter the extent of phosphorylation of the NR2B found in the TIF.

Our results differ from recent data from Nash et al. (2005) showing modifications of total striatal levels of PSD-95 and SAP97 in parkinsonism and L-DOPAinduced dyskinesia. These apparent discrepancies can be related to the different lesioning paradigms and L-DOPA treatment as well as different subcellular fractionation-solubilization techniques used (Nash et al., 2005). In fact, whereas our biochemical experiments are performed in a TIF (Gardoni et al., 2001) highly enriched in PSD proteins, none of the previous studies used such a high detergent extraction protocol to obtain a fraction in which the NMDA signaling complex is enriched. These experimental differences could also explain the absence of any modification of NR2B tyrosine phosphorylation found in our TIF preparation compared with previous studies (Menegoz et al., 1995; Dunah et al., 2000).

The protein levels of any component of the NMDA receptor complex (NMDA subunits and MAGUK elements) present in total striatal homogenates were not modified in the 6-OHDA-lesioned and L-DOPA-treated rats. These data are in agreement with previous studies (Menegoz et al., 1995; Dunah et al., 2000; Hallett et al., 2005), confirming the idea that a subcellular redistribution and not an altered expression of key elements of the NMDA receptor complex could represent the main event in striatal neurons in experimental parkinsonism.

It is well known that, on the surface of living neurons, there are mobile and immobile pools of NMDA receptors at both synaptic and extrasynaptic sites (Tovar and Westbrook, 2002). From a functional point of view, synaptic NMDA complexes, containing the NR2B subunits, promote nuclear signaling to CREB, whereas
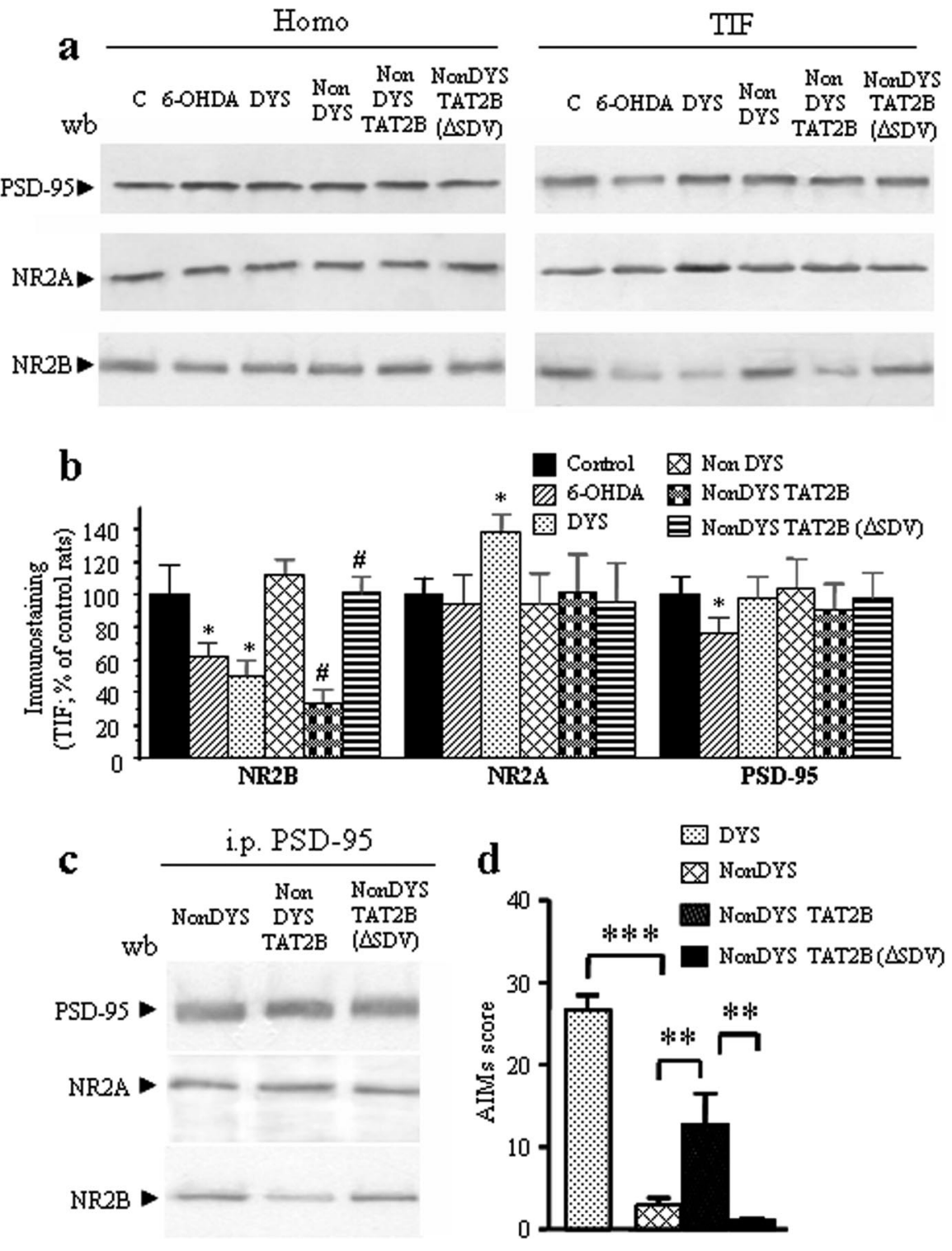

Figure 7. a, Striatal homogenates (Homo; left) and TIF (right) from control (C), 6-OHDA-lesioned, dyskinetic (DYS), nondyskinetic (Non DYS), nondyskinesia plus TAT2B (Non DYS TAT2B), and nondyskinesia plus TAT2B( $\Delta$ SDV) [NonDYS TAT2B( $\triangle$ SDV)] animals were analyzed by Western blot (wb) analysis with PSD-95, NR2A, and NR2B antibodies. The same amount of protein was loaded per lane ( $n=6$ in each group). $\boldsymbol{b}$, Histogram shows quantification of NR2B, NR2A, and PSD-95 in the striatal TIF in the various experimental groups [ ${ }^{*} p<0.05$, NR2B 6-0HDA vs control and dyskinesia vs control; ${ }^{\#} p<0.01$, NR2B nondyskinesia plus TAT2B vs nondyskinesia and nondyskinesia plus TAT2B( $\triangle$ SDV) vs nondyskinesia plus TAT2B; ${ }^{*} p<0.05$, NR2A dyskinesia vs control; ${ }^{*} p<0.05$, PSD-95 6-OHDA vs control; $n=6$ in each group]. $c$, Coimmunoprecipitation of PSD-95 with NR2A and NR2B subunit in homogenate from nondyskinetic animals in the absence or presence of TAT2B (500 $\mu \mathrm{M})$ or TAT2B $(\Delta \mathrm{SDV})(500 \mu \mathrm{M})$. TAT2B peptide reduced the optical density of NR2B but not NR2A subunit in the PSD-95 immunocomplex when compared with untreated rats ( $n=6$ in each group). No effect was induced by the TAT2B $(\Delta S D V)$ peptide. $\boldsymbol{d}$, To analyze the effect of TAT2B injection on the L-DOPA-induced dyskinesias of nondyskinetic rats, rating of AIMs were performed each day of peptides injection 20 min after L-DOPA administration. Three consecutive AIM tests before TAT2B injection were compared with the last three AIM ratings performed after each injection. Chronic dissociation of the MAGUK-NR2B subunit interaction through the injections of TAT2B coupled to L-DOPA administration showed the induction of AIMs in nondyskinetic rats $\left[{ }^{* *} p<0.05\right.$, nondyskinesia vs nondyskinesia plus TAT2B and nondyskinesia plus TAT2B $(\Delta S D V)$ vs nondyskinesia plus TAT2B; ${ }^{* *} p<0.01$ dyskinesia vs nondyskinesia; $n=6$ in each group].

extrasynaptic complexes antagonize nuclear signaling to CREB. Thus, the biological consequences of NMDA receptor activation are a direct consequence of the location of the NMDA receptor signaling complex activated (Hardingham et al., 2002). Results presented here confirm and strengthen the functional role of correct NR2B-CREB cascade inside striatal neurons and propose that a reversible modification of this pathway is involved in both 
experimental parkinsonism and neuronal response to chronic L-DOPA.

Recent studies in primates suggested that upregulation of NR2A abundance in synaptosomal membranes may be an important player in L-DOPA-induced dyskinesias (Hallett et al., 2005). Accordingly, we show here that NR2A synaptic levels are increased in dyskinetic rats, confirming the idea that an overall modification of NMDA receptors at synaptic sites could be considered important for dyskinetic motor behavior. Conversely, experiments performed here with TAT2B peptide clearly demonstrate that abnormal NR2B redistribution between synaptic and extrasynaptic membranes represents an important molecular disturbance of the glutamatergic synapse involved in L-DOPAinduced dyskinesia.

Previous findings demonstrated that peptides analogous to the C-terminal domains of NR2B did not show any effect on basal synaptic transmission or induction of long-term potentiation in hippocampal slices (Lim et al., 2003) even if it produced a decrease in the number of dendritic clusters of PSD-95 and NMDA receptors, suggesting some role of PSD-95 and its homologs in NMDA receptor clustering at synapses. Interestingly, the same peptide used in the present study was described to be able to reduce in vitro NMDA-induced cell death and in vivo ischemia (Aarts et al., 2002). Our data confirm all of these findings and confirm the critical role of a specific in vivo action on NMDA receptor complex by disrupting NR2B binding to MAGUK proteins and, consequently, subcellular localization of the receptor subunit.

Recently, two studies described apparently conflicting results on the effects of the NR2B-selective NMDA receptor antagonist CP-101,606 [(1S,2S)-1-(4-hydroxyphenyl)-2-(4-hydroxy-4phenylpiperidino)-1-propanol] on L-DOPA-induced dyskinesia in two different models of experimental parkinsonism (Nash et al., 2004; Wessell et al., 2004). However, they agreed in identifying NR2B as a key element in either experimental parkinsonism or the development of L-DOPA-induced dyskinesia (Nash et al., 2000; Hadj Tahar et al., 2004; Loschmann et al., 2004).

Although the role of the MAGUK protein family has been extensively studied in cultured neurons, their functions in vivo are still not well established. In particular, the in vivo importance of SAP97 and SAP102 for brain function is unclear, although in vitro studies showed their involvement in NMDA receptor trafficking (Kim and Sheng, 2004). Here we add relevant knowledge on the role of these MAGUK proteins in driving a physiological motor response modulating in vivo NMDA receptor subunit localization and function, showing that a modification of MAGUK binding to a specific subunit of NMDA receptor is strictly correlated to abnormal motor behavior after chronic L-DOPA treatment (supplemental Fig. 1, available at www.jneurosci.org as supplemental material).

\section{References}

Aarts M, Liu Y, Liu L, Besshoh S, Arundine M, Gurd JW, Wang YT, Salter MW, Tymianski M (2002) Treatment of ischemic brain damage by perturbing NMDA receptor-PSD-95 protein interactions. Science 298:846-850

Cenci MA, Lee CS, Björklund A (1998) L-DOPA-induced dyskinesia in the rat is associated with striatal overexpression of prodynorphin- and glutamic acid decarboxylase mRNA. Eur J Neurosci 10:2694-2706.

Dunah AW, Standaert DG (2001) Dopamine $\mathrm{D}_{1}$ receptor-dependent trafficking of striatal NMDA glutamate receptors to the postsynaptic membrane. J Neurosci 21:5546-5558.

Dunah AW, Wang Y, Yasuda RP, Kameyama K, Huganir RL, Wolfe BB, Standaert DG (2000) Alterations in subunit expression, composition, and phosphorylation of striatal $\mathrm{N}$-methyl-D-aspartate glutamate recep- tors in a rat 6-hydroxydopamine model of Parkinson's disease. Mol Pharmacol 57:342-352.

Gardoni F, Caputi A, Cimino M, Pastorino L, Cattabeni F, Di Luca M (1998) Calcium/calmodulin-dependent protein kinase II is associated with NR2A/B subunits of NMDA receptor in postsynaptic densities. J Neurochem 71:1733-1741.

Gardoni F, Schrama LH, Kamal A, Gispen WH, Cattabeni F, Di Luca M (2001) Hippocampal synaptic plasticity involves competition between $\mathrm{Ca}^{2+} /$ calmodulin-dependent protein kinase II and postsynaptic density 95 for binding to the NR2A subunit of the NMDA receptor. J Neurosci 21:1501-1509.

Gardoni F, Mauceri D, Fiorentini C, Bellone C, Missale C, Cattabeni F, Di Luca M (2003a) CaMKII-dependent phosphorylation regulates SAP97/ NR2A interaction. J Biol Chem 278:44745-44752.

Gardoni F, Pagliardini S, Setola V, Bassanini S, Cattabeni F, Battaglia G, Di Luca M (2003b) The NMDA receptor complex is altered in an animal model of human cerebral heterotopia. J Neuropathol Exp Neurol 62:662-675.

Hadj Tahar A, Gregoire L, Darre A, Belanger N, Meltzer L, Bedard PJ (2004) Effect of a selective glutamate antagonist on L-dopa-induced dyskinesias in drug-naive parkinsonian monkeys. Neurobiol Dis 15:171-176.

Hallett PJ, Dunah AW, Ravenscroft P, Zhou S, Bezard E, Crossman AR, Brotchie JM, Standaert DG (2005) Alterations of striatal NMDA receptor subunits associated with the development of dyskinesia in the MPTPlesioned primate model of Parkinson's disease. Neuropharmacology 48: 503-516.

Hardingham GE, Fukunaga Y, Bading H (2002) Extrasynaptic NMDARs oppose synaptic NMDARs by triggering CREB shut-off and cell death pathways. Nat Neurosci 5:405-414.

Kim E, Sheng M (2004) PDZ domain proteins of synapses. Nat Rev Neurosci 5:771-781.

Lang AP, Lozano AE (1998) Parkinson's disease. N Engl J Med 339:1044-1053.

Lim IA, Merrill MA, Chen Y, Hell JW (2003) Disruption of the NMDA receptor-PSD-95 interaction in hippocampal neurons with no obvious physiological short-term effect. Neuropharmacology 45:738-754.

Loschmann PA, De Groote C, Smith L, Wullner U, Fischer G, Kemp JA, Jenner P, Klockgether T (2004) Antiparkinsonian activity of Ro 25-6981, a NR2B subunit specific NMDA receptor antagonist, in animal models of Parkinson's disease. Exp Neurol 187:86-93.

Lundblad M, Andersson M, Winkler C, Kirik D, Wierup N, Cenci MA (2002) Pharmacological validation of behavioural measures of akinesia and dyskinesia in a rat model of Parkinson's disease. Eur J Neurosci 15:120-132.

Menegoz M, Lau LF, Herve D, Huganir RL, Girault JA (1995) Tyrosine phosphorylation of NMDA receptor in rat striatum: effects of 6-OHdopamine lesions. NeuroReport 7:125-128.

Nash JE, Fox SH, Henry B, Hill MP, Peggs D, McGuire S, Maneuf Y, Hille C, Brotchie JM, Crossman AR (2000) Antiparkinsonian actions of ifenprodil in the MPTP-lesioned marmoset model of Parkinson's disease. Exp Neurol 165:136-142.

Nash JE, Ravenscroft P, McGuire S, Crossman AR, Menniti FS, Brotchie JM (2004) The NR2B-selective NMDA receptor antagonist CP-101,606 exacerbates L-DOPA-induced dyskinesia and provides mild potentiation of anti-parkinsonian effects of L-DOPA in the MPTP-lesioned marmoset model of Parkinson's disease. Exp Neurol 188:471-479.

Nash JE, Johnston GL, Collingridge GL, Garner CC, Brotchie JM (2005) Subcellular redistribution of the synapse-associated proteins PSD-95 and SAP97 in animal models of Parkinson's disease and L-DOPA-induced dyskinesia. FASEB J 19:583-585.

Obeso JA, Olanow CW, Nutt JC (2000) Levodopa motor complications in Parkinson's disease. Trends Neurosci 23:S2-S7.

Oh JD, Vaughan CL, Chase TN (1999) Effect of dopamine denervation and dopamine agonist administration on serine phosphorylation of striatal NMDA receptor subunits. Brain Res 821:433-442.

Omkumar RV, Kiely MJ, Rosenstein AJ, Min KT, Kennedy MB (1996) Identification of a phosphorylation site for calcium/calmodulindependent protein kinase II in the NR2B subunit of the $N$-methyl-D-aspartate receptor. J Biol Chem 271:31670-31678.

Paxinos G, Watson C (1986) The rat brain in stereotaxic coordinates. Orlando, FL: Academic. 
Picconi B, Centonze D, Hakansson K, Bernardi G, Greengard P, Fisone G, Cenci MA, Calabresi P (2003) Loss of bidirectional striatal synaptic plasticity in L-DOPA-induced dyskinesia. Nat Neurosci 6:501-506.

Picconi B, Gardoni F, Centonze D, Mauceri D, Cenci MA, Bernardi G, Calabresi P, Di Luca M (2004) Abnormal $\mathrm{Ca}^{2+}$-calmodulin-dependent protein kinase II function mediates synaptic and motor deficits in experimental parkinsonism. J Neurosci 24:5283-5291.

Prybylowski K, Wenthold RJ (2004) N-Methyl-D-aspartate receptors: subunit assembly and trafficking to the synapse. J Biol Chem 279:9673-9676.

Sans N, Prybylowski K, Petralia RS, Chang K, Wang YX, Racca C, Vicini S, Wenthold RJ (2003) NMDA receptor trafficking through an interaction between PDZ proteins and the exocyst complex. Nat Cell Biol 5:520-530. Schwarting RK, Huston JP (1996) The unilateral 6-hydroxydopamine le- sion model in behavioral brain research. Analysis of functional deficits, recovery and treatments. Prog Neurobiol 50:275-331.

Sheng M (2001) Molecular organization of the postsynaptic specialization. Proc Natl Acad Sci USA 98:7058-7061.

Strack S, Colbran RJ (1998) Autophosphorylation-dependent targeting of calcium/ calmodulin-dependent protein kinase II by the NR2B subunit of the $N$-methyl-D-aspartate receptor. J Biol Chem 273:20689-20692.

Tovar KR, Westbrook GL (2002) Mobile NMDA receptors at hippocampal synapses. Neuron 34:255-264.

Wessell RH, Ahmed SM, Menniti FS, Dunbar GL, Chase TN, Oh JD (2004) NR2B selective NMDA receptor antagonist CP-101,606 prevents levodopa-induced motor response alterations in hemi-parkinsonian rats. Neuropharmacology 47:184-194. 